\title{
CONJUGATE SYSTEMS WITH EQUAL TANGENTIAL INVARIANTS AND THE TRANSFORMATION OF MOUTARD.
}

\author{
By Luther Pfahler Eisenhart (Princeton, N. J.).
}

Adunanza del 12 aprile 1914.

In recent years one of the most interesting fields of geometrical investigations has been that of transformations of geometrical entities of a particular type into entities of the same type. As examples, we mention the well-known Bäcklund transformations of pseudospherical surfaces, the Bianchi transformations $B_{k}$ of surfaces applicable to quadrics ${ }^{\mathbf{I}}$ ), and the transformation of the surfaces of Voss ${ }^{2}$ ). In the first two cases a surface $S$ and a transform $S_{i}$ are the focal surfaces of a $W$-congruence. Bianchi ${ }^{3}$ ) has shown the analytic relation between $W$-congruences and the transformation of Moutard ${ }^{4}$ ) and consequently we have general methods of procedure in the determination of any transformation for which the two surfaces are the focal surfaces of a $W$-congruence. Moreover, it should be said that there are other known transformations of this type than those mentioned above. In the third example referred to a surface $S$ and a transform $S_{1}$ are so related that the lines joining corresponding points on their surfaces form a congruence whose developables meet $S$ and $S_{\mathrm{I}}$ in a conjugate system with equal tangential invariants; namely the geodesic conjugate system. It is the purpose of this paper to establish for any conjugate system with equal tangential invariants a transformation possessing the property just mentioned and determine other properties of this transformation.

When a surface $S$ is referred to a conjugate system with equal tangential invariants, one can find by quadratures an associate surface $\Sigma$ referred to its asymptotic lines. For the transformation under discussion the associate surfaces $\mathbf{\Sigma}$ and $\mathbf{\Sigma}_{\mathrm{t}}$ of $S$ and $S_{\mathrm{t}}$

I) L. Bianchi, Lezioni di Geometria differenziale, Vol. III: Teoria delle trasformazioni delle superficie applicabili sulle quadriche (Pisa, Spoerri, 1909).

2) L. P. Eisenhart, Transformations of Surfaces of Voss [Transactions of the American Mathematical Society, Vol. XV (Igr4), pp. 24,5-265].

3) Bianchi, Lezioni di Geometria differenziale, Vol. II (Pisa, Spoerri, 1903), pp. 5I-56.

4) TH. F. Moutard, Sur la construction des équations de la forme $\frac{\mathrm{I}}{z} \frac{\partial^{2} z}{\partial x \partial y}=\lambda(x, y)$ qui admettent une intégrale gentrale explicite [Journal de l'École Polytechnique, Series I, Cahier XLV (I878), pp. I-II]; also: L. BIanchI, Lęioni di Geometria differenziale, Vol. II (Pisa, Spoerri, r903), pp. 47, 48. 
respectively are the focal sheets of a $W$-congruence. Accordingly we devote $\$ \int I, 2$ to the determination in suitable form of the equations of a $W$-congruence. With the aid of these results we establish in $\ 3$ the equations of the desired transformations $\mathbf{\Omega}$ of the given surface $S$.

In $\ 4$ we show that these general transformations possess a theorem of permutavility, that is, if $S_{1}$ and $S_{2}$ are transforms of $S$, there exist $\infty^{1}$ surfaces $S^{\prime}$ each of which is obtained from $S_{1}$ and $S_{2}$ by transformations $\Omega$. Moreover, if $M, M_{1}, M_{2}$ are corresponding points of $S, S_{1}, S_{2}$ the corresponding points $M^{\prime}$ of the surfaces $S^{\prime}$ lie on a conic in the plane of $M, M_{1}, M_{2}$; and the tangent planes to the surfaces $S^{\prime}$ pass through the point of intersection of the corresponding tangent planes to $S, S_{1}$ and $S_{2}$.

In $\ S$ we consider the congruence of lines of intersection of the tangent planes to a surface $S$ and a transform $S_{1}$, and in $\$ 6$ discuss the case for which this congruence is normal. To this class belong the transformations of surfaces of Voss previously referred to ${ }^{5}$ ).

In the last section we determine under what conditions $S$ and a transform $S_{\mathrm{I}}$ envelope a two parameter family of spheres and find that the corresponding conjugate system of $S$ and $S_{1}$ are lines of curvature with isothermal spherical representation. This is the transformation which we established sometime since proceeding from purely analytical considerations.

\section{$\int I$.}

\section{Equations of a non-normal $W$-congruence.}

I. When a surface $\Sigma$ is referred to its asymptotic lines and the linear element of the spherical representation of these lines is written

$$
d \sigma^{2}=E d u^{2}+2 \sqrt{E G} \cos 2 \omega d u d v+G d v^{2},
$$

$2 \omega$ is the angle between the parametric lines on the sphere.

If $\left\{\begin{array}{c}\mathrm{I} 2 \\ \mathrm{I}\end{array}\right\}$ and $\left\{\begin{array}{c}\mathrm{I} 2 \\ 2\end{array}\right\}$ are Christoffel symbols formed with respect to this linear element, and the total curvature of $\Sigma$ be denoted by $-\frac{1}{p^{2}}$, we have

$$
\left.\left\{\begin{array}{c}
\mathrm{I} 2 \\
\mathrm{I}
\end{array}\right\}^{\prime}=-\frac{\mathrm{I}}{2} \frac{\partial \log \rho}{\partial v}, \quad\left\{\begin{array}{c}
\mathrm{I} 2 \\
2
\end{array}\right\}^{\prime}=-\frac{\mathrm{I}}{2} \frac{\partial \log \rho}{\partial u}{ }^{6}\right) .
$$

Moreover, from the definition of the symbols $\left\{\begin{array}{c}r s \\ t\end{array}\right\}$ and equations (2) follow the iden-

5) Loc. cit. $\left.{ }^{2}\right)$.

6) L. P. Eisenhart, A Treatise on the Differential Geometry of Curves and Surfaces (London and Boston, Ginn and Co., rgog), p. rg2. 
tities

$$
\left\{\begin{array}{l}
\frac{\partial \sqrt{E}}{\partial v}=-\sqrt{E} \frac{\partial \log \sqrt{\rho}}{\partial v}-\sqrt{G} \cos 2 \omega \frac{\partial \log \sqrt{\rho}}{\partial u}, \\
\frac{\partial \sqrt{G}}{\partial u}=-\sqrt{G} \frac{\partial \log \sqrt{\rho}}{\partial u}-\sqrt{E} \cos 2 \omega \frac{\partial \log \sqrt{\rho}}{\partial v} .
\end{array}\right.
$$

If $X, Y, Z$ denote the direction-cosines of the normal to $\Sigma$ and $X_{1}, Y_{1}^{\circ}, Z_{1}$; $X_{2}, Y_{2}, Z_{2}$ the direction-cosines of the bisectors of the angles between the parametric lines on the spherical representation of $\Sigma$, we have

(4)

$$
\begin{cases}\frac{\partial X}{\partial u}=\sqrt{E}\left(\sin \omega X_{\mathrm{r}}+\cos \omega X_{2}\right), & \frac{\partial X}{\partial v}=\sqrt{G}\left(-\sin \omega X_{\mathrm{r}}+\cos \omega X_{2}\right), \\ \frac{\partial X_{\mathrm{r}}}{\partial u}=-A X_{2}-\sqrt{E} \sin \omega X, & \frac{\partial X_{1}}{\partial v}=B X_{2}+\sqrt{G} \sin \omega X \\ \frac{\partial X_{2}}{\partial u}=A X_{\mathrm{r}}-\sqrt{E} \cos \omega X, & \frac{\partial X_{2}}{\partial v}=-B X_{\mathrm{r}}-\sqrt{G} \cos \omega X\end{cases}
$$

where

(5) $A=\frac{\partial \omega}{\partial u}-\sqrt{\frac{E}{G}} \sin 2 \omega \frac{\partial \log \sqrt{\rho}}{\partial v}, \quad B=\frac{\partial \omega}{\partial v}-\sqrt{\frac{G}{E}} \sin 2 \omega \frac{\partial \log \sqrt{\rho}}{\partial u}$.

If we make use of the Liouville form of the Gaussian curvature ${ }^{7}$ ), we can show that

$$
\frac{\partial \cdot A}{\partial v}+\frac{\partial B}{\partial u}+\sqrt{E G} \sin 2 \omega=0 .
$$

In accordance with the general theory we know that $X, Y, Z$ are solutions of

$$
\frac{\partial^{2} \theta}{\partial u \partial v}+\frac{\partial \log \sqrt{\rho}}{\partial v} \frac{\partial \theta}{\partial u}+\frac{\partial \log \sqrt{\rho}}{\partial u} \frac{\partial \theta}{\partial v}+\sqrt{E G} \cos 2 \omega \cdot \theta=0 .
$$

If $\Sigma_{\mathrm{r}}$ is a surface which together with $\mathbf{\Sigma}$ constitutes the focal sheets of a $W$-congruence, the direction-cosines of the join of corresponding points may be written in the form

(8) $\alpha_{1}=\cos \theta X_{1}+\sin \theta X_{2}, \quad \beta_{1}=\cos \theta Y_{1}+\sin \theta Y_{2}, \quad \gamma_{1}=\cos \theta Z_{1}+\sin \theta Z_{2}$.

If $\sigma$ denotes the angle between the tangent planes to $\Sigma$ and $\Sigma_{1}$, and $X^{\prime}, Y^{\prime}, Z^{\prime}$, the direction-cosines of the normal to $\Sigma_{1}$, we have

$$
X^{\prime}=\cos \sigma X+\sin \sigma\left(\sin \theta X_{1}-\cos \theta X_{2}\right) \text {. }
$$


From this we derive, making use of (4),

(10)

$$
\left\{\begin{array}{c}
\frac{\partial X^{\prime}}{\partial u}=-\sin \sigma\left[\frac{\partial \sigma}{\partial u}-\sqrt{E} \cos (\theta+\omega)\right] X \\
+\left[\cos \sigma\left(\sqrt{E} \sin \omega+\sin \theta \frac{\partial \sigma}{\partial u}\right)+\sin \sigma \cos \theta\left(\frac{\partial \theta}{\partial u}-A\right)\right] X_{1} \\
+\left[\cos \sigma\left(\sqrt{E} \cos \omega-\cos \theta \frac{\partial \sigma}{\partial u}\right)+\sin \sigma \sin \theta\left(\frac{\partial \theta}{\partial u}-A\right)\right] X_{2} \\
\frac{\partial X^{\prime}}{\partial v}=-\sin \sigma\left[\frac{\partial \sigma}{\partial v}-\sqrt{G} \cos (\theta-\omega)\right] X \\
+\left[\cos \sigma\left(-\sqrt{G} \sin \omega+\sin \theta \frac{\partial \sigma}{\partial v}\right)+\sin \sigma \cos \theta\left(\frac{\partial \theta}{\partial v}+B\right)\right] X_{1} \\
+\left[\cos \sigma\left(\sqrt{G} \cos \omega-\cos \theta \frac{\partial \sigma}{\partial v}\right)+\sin \sigma \sin \theta\left(\frac{\partial \theta}{\partial v}+B\right)\right] X_{2} .
\end{array}\right.
$$

The direction-cosines $X^{\prime}, Y^{\prime}, Z^{\prime}$ are given by the quadratures

(I I) $\frac{\partial}{\partial u}\left(\sqrt{\rho p_{1}} w_{1} X^{\prime}\right)=-\rho w_{1}^{2} \frac{\partial}{\partial u}\left(\frac{X}{w_{1}}\right), \quad \frac{\partial}{\partial v}\left(\sqrt{\rho p_{1}} w_{1} X^{\prime}\right)=\rho w_{1}^{2} \frac{\partial}{\partial v}\left(\frac{X}{w_{1}}\right)$.

where now $w_{1}$ is a solution of the equation $(7)^{8}$ ).

The cartesian coordinates $\xi_{1}, n_{1}, \zeta_{1}$, of $\Sigma_{1}$ are given by equations of the form

$$
\left.\zeta_{\mathrm{I}}-\xi=\sqrt{\rho \rho_{1}}\left(Y Z^{\prime}-Z Y^{\prime}\right)=\sqrt{\rho \rho_{1}} \sin \sigma\left(\cos \theta X_{1}+\sin \theta X_{2}\right)^{9}\right) \text {. }
$$

Also we have

(13) $\frac{\partial \xi}{\partial \imath}=\sqrt{E} \bar{E}_{p}\left(\cos \omega X_{1}-\sin \omega X_{2}\right), \quad \frac{\partial \xi}{\partial v}=-\sqrt{G} p\left(\cos \omega X_{1}+\sin \omega X_{2}\right)$, and

$$
\mathbb{E}=E \rho^{2}, \quad \mathfrak{F}=-\rho^{2} \sqrt{E G} \cos 2 \omega, \quad \mathscr{H}=G \rho^{2},
$$

where $E, F$ and $G$ denote the first fundamental coefficients of $\Sigma$.

If in (II) we replace $\frac{\partial X}{\partial u}$ and $\frac{\partial X}{\partial v}$ by their values from (4) we have

$$
\left\{\begin{array}{l}
\frac{\partial X^{\prime}}{\partial u}=-\frac{\partial}{\partial u} \log \left(\sqrt{\rho_{\rho_{1}}} w_{1}\right) X^{\prime}-\sqrt{\frac{\rho}{\rho_{1}}}\left[\sqrt{E}\left(\sin \omega X_{1}+\cos \omega X_{2}\right)-X \frac{\partial \log w_{1}}{\partial u}\right] \\
\frac{\partial X^{\prime}}{\partial v}=-\frac{\partial}{\partial v} \log \left(\sqrt{\rho_{\rho_{1}}} w_{1}\right) X^{\prime}-\sqrt{\frac{\rho}{\rho_{1}}}\left[\sqrt{G}\left(\sin \omega X_{1}-\cos \omega X_{2}\right)+X \frac{\partial \log w_{1}}{\partial v}\right] .
\end{array}\right.
$$

Equating these expressions to the values given by (10), we obtain the following

8) This follows from equations (74) [loc. cit. ${ }^{6}$ ), p. 419] where we put

$$
\begin{aligned}
& v_{1}=\sqrt{\rho} X, \quad v_{2}=\sqrt{\rho} Y, \quad v_{3}=\sqrt{\rho} Z ; \quad \overline{v_{1}}=\sqrt{\rho_{1}} X^{\prime}, \quad \overline{v_{2}}=\sqrt{\rho_{1}} Y^{\prime}, \quad \overline{v_{3}}=\sqrt{\rho_{1}} Z^{\prime} ; \quad \theta_{1}=\sqrt{\rho} \omega_{1} . \\
& \text { 9) Loc. cit. }{ }^{6} \text { ), p. } 4 \text { 18. }
\end{aligned}
$$


equations:

$$
\left\{\begin{array}{c}
\sin \sigma\left[\frac{\partial \sigma}{\partial u}-\sqrt{E} \cos (\theta+\omega)\right]=\cos \sigma \frac{\partial \log \sqrt{\rho_{1}}}{\partial u}+\left(\cos \sigma-\sqrt{\frac{\rho}{\rho_{1}}}\right) \frac{\partial \log w_{1}}{\partial u} \\
\sin \sigma\left[\frac{\partial \sigma}{\partial v}-\sqrt{G} \cos (\theta-\omega)\right]=\cos \sigma \frac{\partial \log \sqrt{\rho \rho_{1}}}{\partial v}+\left(\cos \sigma+\sqrt{\frac{\rho}{\rho_{1}}}\right) \frac{\partial \log w_{1}}{\partial v} \\
\cos \sigma \frac{\partial \sigma}{\partial u}+\frac{\partial}{\partial v} \log \left(\sqrt{\rho \rho_{1}} w_{1}\right) \cdot \sin \sigma-\sqrt{E} \cos (\theta+\omega)\left(\cos \sigma+\sqrt{\frac{\rho}{\rho_{1}}}\right)=0 \\
\cos \sigma \frac{\partial \sigma}{\partial v}+\frac{\partial}{\partial v} \log \left(\sqrt{\rho \rho_{1}} w_{1}\right) \cdot \sin \sigma-\sqrt{G} \cos (\theta-\omega)\left(\cos \sigma-\sqrt{\frac{\rho}{\rho_{1}}}\right)=0 \\
\sin \sigma\left(\frac{\partial \theta}{\partial u}-A\right)+\sqrt{E}\left(\cos \sigma+\sqrt{\frac{\rho}{\rho_{1}}}\right) \sin (\theta+\omega)=0 \\
\sin \sigma\left(\frac{\partial \theta}{\partial v}+B\right)+\sqrt{G}\left(\cos \sigma-\sqrt{\frac{\rho}{\rho_{1}}}\right) \sin (\theta-\omega)=0 .
\end{array}\right.
$$

For the present we exclude the case where $\sigma=\frac{\pi}{2}$ and thus equations (16) are equivalent to

$$
\left\{\begin{array}{l}
\frac{\partial \sigma}{\partial u}=\sqrt{E} \cos (\theta+\omega)\left(I+\cos \sigma \sqrt{\frac{\rho}{\rho_{I}}}\right)-\sqrt{\frac{\rho}{\rho_{I}}} \sin \sigma \frac{\partial \log w_{I}}{\partial u}, \\
\frac{\partial \sigma}{\partial v}=\sqrt{G} \cos (\theta-\omega)\left(I-\cos \sigma \sqrt{\frac{\rho}{\rho_{1}}}\right)+\sqrt{\frac{\rho_{1}}{\rho_{I}}} \sin \sigma \frac{\partial \log w_{I}}{\partial v},
\end{array}\right.
$$

$$
\begin{gathered}
\left\{\begin{array}{l}
\frac{\partial \log \sqrt{\rho_{P_{I}}}}{\partial u}+\frac{\partial \log w_{1}}{\partial u}\left(I-\sqrt{\frac{\rho}{\rho_{I}}} \cos \sigma\right)-\sqrt{E} \sin \sigma \sqrt{\frac{\rho}{P_{I}}} \cos (\theta+\omega)=0, \\
\frac{\partial \log \sqrt{\rho_{P_{I}}}}{\partial v}+\frac{\partial \log w_{1}}{\partial v}\left(I+\sqrt{\frac{\rho}{\rho_{I}}} \cos \sigma\right)+\sqrt{G} \sin \sigma \sqrt{\frac{\rho}{\rho_{I}}} \cos (\theta-\omega)=0,
\end{array}\right. \\
\left\{\begin{array}{l}
\sin \sigma\left(\frac{\partial \theta}{\partial u}-A\right)+\sqrt{E}\left(\cos \sigma+\sqrt{\frac{\rho}{\rho_{I}}}\right) \sin (\theta+\omega)=0, \\
\sin \sigma\left(\frac{\partial \theta}{\partial v}+B\right)+\sqrt{G}\left(\cos \sigma-\sqrt{\frac{\rho}{\rho_{I}}}\right) \sin (\theta-\omega)=0 .
\end{array}\right.
\end{gathered}
$$

It can readily be shown that the conditions of integrability of these equations are satisfied ${ }^{10}$ ).

10) Equations (17), (18), (19) are essentially the same as those found by M. Picone, Sulle congruence rettilinee $W$ [Rendiconti del Circolo Matematico di Palermo, t. XXXVII ( ${ }^{\circ}$ semestre I9I4), pp. 212-244], p. 220. We noticed this work of PICONE as we were preparing our results for publication. Our method makes use of the transformation of Moutard and thereby is much shorter. These equations and the following ones of this section are extremely useful in discussing particular types of $W$-congruences. 
2. With the aid of equations (I7), (I8), (I9) equations (10) are reducible to

(20)

$$
\left\{\begin{array}{c}
\frac{\partial X^{\prime}}{\partial u}=\sqrt{\frac{\rho}{p_{1}}}\left\{\left[X \sin \sigma-\cos \sigma\left(\sin \theta X_{1}-\cos \theta X_{2}\right)\right]\left[\frac{\partial \log w_{1}}{\partial u} \sin \sigma-\sqrt{E} \cos \sigma \cos (\theta+\omega)\right]\right. \\
\left.-\sqrt{E} \sin (\theta+\omega)\left(X_{1} \cos \theta+X_{2} \sin \theta\right)\right\}, \\
\frac{\partial X^{\prime}}{\partial v}=\sqrt{\frac{\rho}{\rho_{1}}}\left\{\left[X \sin \sigma-\cos \tau\left(\sin \theta X_{1}-\cos \theta X_{2}\right)\right]\left[-\frac{\partial \log w_{1}}{\partial v} \sin \sigma+\sqrt{G} \cos \tau \cos (\theta-\omega)\right]\right. \\
\left.+\sqrt{G} \sin (\theta-\omega)\left(X_{1} \cos \theta+X_{2} \sin \theta\right)\right\} .
\end{array}\right.
$$

We introduce functions $\theta_{1} ; X_{1}^{\prime}, Y_{1}^{\prime}, Z_{1}^{\prime} ; X_{2}^{\prime}, Y_{2}^{\prime}, Z_{2}^{\prime}$ defined by

$$
\left\{\begin{array}{c}
\cos \theta_{1} X_{1}^{\prime}+\sin \theta_{1} X_{2}^{\prime}=\cos \theta X_{1}+\sin \theta X_{2}, \\
-\sin \theta_{1} X_{1}^{\prime}+\cos \theta_{1} X_{2}^{\prime}=X \sin \sigma-\cos \sigma\left(\sin \theta X_{1}-\cos \theta X_{2}\right),
\end{array}\right.
$$

and analogous equations in the $Y^{\prime} s$ and $Z^{\prime} s$.

It follows from (9) and (2I) that

$$
\left|\begin{array}{lll}
X^{\prime} & Y^{\prime} & Z^{\prime} \\
X_{1}^{\prime} & Y_{1}^{\prime} & Z_{\mathrm{r}}^{\prime} \\
X_{2}^{\prime} & Y_{2}^{\prime} & Z_{2}^{\prime}
\end{array}\right|=+\mathrm{I} .
$$

From (2I) follow also
(23)
$\left\{\begin{array}{l}X_{1}^{\prime}=\cos \theta_{1}\left[\cos \theta X_{1}+\sin \theta X_{2}\right]-\sin \theta_{1}\left[X \sin \sigma-\cos \theta\left(\sin \theta X_{1}-\cos \theta X_{2}\right)\right] \\ X_{2}^{\prime}=\sin \theta_{1}\left[\cos \theta X_{1}+\sin \theta X_{2}\right]+\cos \theta_{1}\left[X \sin \sigma-\cos \sigma\left(\sin \theta X_{1}-\cos \theta X_{2}\right)\right] .\end{array}\right.$

We denote by $\omega_{1}, E_{1}, G_{1}$ functions for $\Sigma_{1}$ analogous to $\omega, E, G$ for $\mathbf{\Sigma}$. We imagine $\theta_{1}$ determined so that we hawe

(24) $\frac{\partial X^{\prime}}{\partial u}=\sqrt{E_{\mathrm{r}}}\left(\sin \omega_{\mathrm{r}} X_{\mathrm{r}}^{\prime}+\cos \omega_{1} X_{2}^{\prime}\right), \quad \frac{\partial X^{\prime}}{\partial v}=\sqrt{G_{1}}\left(-\sin \omega_{\mathrm{t}} X_{\mathrm{s}}^{\prime}+\cos \omega_{1} X_{2}^{\prime}\right)$.

Comparing these with (20), we have

(25)

$$
\begin{aligned}
& \left(\sqrt{E_{1}} \sin \omega_{+}=-\sqrt{\frac{p}{p_{1}}}\left\{\sin \theta_{1}\left[\frac{\partial \log w_{1}}{\partial u} \sin \sigma-\sqrt{E} \cos \sigma \cos (\theta+\omega)\right]+\sqrt{E} \sin (\theta+\omega) \cos \theta_{1}\right\},\right. \\
& \sqrt{E_{1}} \cos \omega_{1}=\sqrt{\frac{\rho}{\rho_{1}}}\left\{\cos \theta_{1}\left[\frac{\partial \log w_{1}}{\partial u} \sin \sigma-\sqrt{E} \cos \sigma \cos (\theta+\omega)\right]-\sqrt{E} \sin (\theta+\omega) \sin \theta_{1}\right\}, \\
& \sqrt{G_{1}} \sin \omega_{1}=-\sqrt{\frac{\rho}{\rho_{1}}}\left\{\sin \theta_{1}\left[\frac{\partial \log w_{1}}{\partial v} \sin \sigma-\sqrt{G} \cos \sigma \cos (\theta-\omega)\right]+\sqrt{G} \sin (\theta-\omega) \cos \theta_{1}\right\}, \\
& \sqrt{ } \bar{G}_{1} \cos \omega=1 \sqrt{\frac{\rho}{\rho_{1}}}\left\{-\cos \theta_{1}\left[\frac{\partial \log w_{1}}{\partial v} \sin \sigma-\sqrt{G} \cos \sigma \cos (\theta-\omega)\right]+\sqrt{G} \sin (\theta-\omega) \sin \theta_{1}\right\} \text {. }
\end{aligned}
$$

These equations are equivalent to

$$
\left\{\begin{array}{l}
\frac{\partial \log w_{1}}{\partial u} \sin \sigma=\sqrt{E}\left[\cos \sigma \cos (\theta+\omega)-\sin (\theta+\omega) \cot \left(\theta_{1}+\omega_{1}\right)\right] \\
\frac{\partial \log w_{1}}{\partial v} \sin \sigma=\sqrt{G}\left[\cos \sigma \cos (\theta-\omega)-\sin (\theta-\omega) \cot \left(\theta_{1}-\omega_{1}\right)\right]
\end{array}\right.
$$


and

(27) $\sqrt{E_{1}}=-\sqrt{\frac{\rho_{1}}{\rho_{1}}} \sqrt{E} \frac{\sin (\theta+\omega)}{\sin \left(\theta_{1}+\omega_{1}\right)}, \quad \sqrt{G_{1}}=\sqrt{\frac{\rho_{1}}{\rho_{1}}} \sqrt{G} \frac{\sin (\theta-\omega)}{\sin \left(\theta_{1}-\omega_{1}\right)}$.

If one calculates $E_{1}$ and $G_{1}$ from (20), it is seen that equations (26) and (27) are consistent as determining $\left(\theta_{1}+\omega_{1}\right)$ and $\left(\theta_{1}-\omega_{1}\right)$. Hence equations $(26)$ may be solved for $\theta_{1}$ as desired.

Now equations (I7) and (I8) are reducible by (26) to

and

$$
\left\{\begin{array}{l}
\frac{\partial \sigma}{\partial u}=\sqrt{E}\left[\cos (\theta+\omega)+\sqrt{\frac{\rho}{\rho_{1}}} \sin (\theta+\omega) \cot \left(\theta_{1}+\omega_{1}\right)\right], \\
\frac{\partial \sigma}{\partial v}=\sqrt{G}\left[\cos (\theta-\omega)-\sqrt{\frac{\rho}{\rho_{1}}} \sin (\theta-\omega) \cot \left(\theta_{1}-\omega_{1}\right)\right],
\end{array}\right.
$$

(29)

$$
\begin{aligned}
& \left(\frac{\partial}{\partial u} \log \sqrt{\rho p_{1}}=\frac{-\sqrt{E}}{\sin \sigma}\left[\left(\cos \sigma-\sqrt{\frac{\rho}{\rho_{1}}}\right) \cos (\theta+\omega)\right.\right. \\
& \left.+\left(1 \sqrt{\frac{\rho}{p_{1}}} \cos \sigma-I\right) \sin (\theta+\omega) \cot \left(\theta_{r}+\omega_{1}\right)\right] \text {, } \\
& \frac{\partial}{\partial v} \log \sqrt{\rho_{\rho_{I}}}=\frac{-\sqrt{G}}{\sin \sigma}\left[\left(\cos \sigma+\sqrt{\frac{\rho}{\rho_{I}}}\right) \cos (\theta-\omega)\right. \\
& \left.-\left(1 \sqrt{\frac{\rho}{\rho_{I}}} \cos \sigma+I\right) \sin (\theta-\omega) \cot \left(\theta_{1}-\omega_{1}\right)\right] \text {. }
\end{aligned}
$$

From these we derive also

$$
\left\{\begin{array}{l}
\frac{\partial}{\partial u} \log \left(\sqrt{\rho \rho_{1}} w_{1}\right)=\frac{\sqrt{E}}{\sin \sigma} \sqrt{\frac{\rho}{\rho_{1}}}\left[\cos (\theta+\omega)-\cos \sigma \sin (\theta+\omega) \cot \left(\theta_{1}+\omega_{1}\right)\right], \\
\frac{\partial}{\partial v} \log \left(\sqrt{\rho \rho_{1}} w_{1}\right)=\frac{\sqrt{G}}{\sin \sigma} \sqrt{\frac{\rho}{\rho_{1}}}\left[-\cos (\theta-\omega)+\cos \sigma \sin (\theta-\omega) \cot \left(\theta_{1}-\omega_{1}\right)\right] .
\end{array}\right.
$$

In view of the fact that $\theta_{1}$ has been chosen so that (24) hold, we must have also, analogous to (4)

$$
\begin{cases}\frac{\partial X_{1}^{\prime}}{\partial u}=-A_{1} X_{2}^{\prime}-\sqrt{E_{1} \sin \omega_{1} X^{\prime},} & \frac{\partial X_{1}^{\prime}}{\partial v}=B_{1} X_{2}^{\prime}+\sqrt{G_{1}} \sin \omega_{1} X^{\prime} \\ \frac{\partial X_{2}^{\prime}}{\partial u}=A_{1} X_{1}^{\prime}-\sqrt{E_{1} \cos \omega_{1} X^{\prime},} & \frac{\partial X_{3}^{\prime}}{\partial v}=-B_{1} X_{1}^{\prime}-\sqrt{G_{1}} \cos \omega_{1} X^{\prime},\end{cases}
$$

where

(32) $A_{\mathrm{I}}=\frac{\partial \omega_{\mathrm{I}}}{\partial u}-\sqrt{\frac{E_{\mathrm{I}}}{G_{\mathrm{I}}}} \sin 2 \omega_{\mathrm{i}} \frac{\partial \log \sqrt{\rho_{\mathrm{I}}}}{\partial v}, \quad B_{\mathrm{s}}=\frac{\partial \omega_{\mathrm{I}}}{\partial v}-\sqrt{\frac{G_{\mathrm{I}}}{E_{\mathrm{I}}}} \sin 2 \omega_{\mathrm{I}} \frac{\partial \log \sqrt{p_{\mathrm{I}}}}{\partial u}$.

In order to find the conditions imposed by this set of equations, we calculate first the derivatives of $\alpha_{1}$ defined by (8) and also of $\alpha_{2}$, where

$$
\alpha_{2}=-\sin \theta X_{1}+\cos \theta X_{2} \text {. }
$$


One finds readily

(34)

$$
\left\{\begin{array}{l}
\frac{\partial \alpha_{1}}{\partial u}=-\sqrt{E} \frac{\sin (\theta+\omega)}{\sin \sigma}\left[\left(\cos \sigma+\sqrt{\frac{\rho}{\rho_{1}}}\right) \alpha_{2}+X \sin \sigma\right], \\
\frac{\partial x_{1}}{\partial v}=-\sqrt{G} \frac{\sin (\theta-\omega)}{\sin \sigma}\left[\left(\cos \sigma-\sqrt{\frac{\rho}{\rho_{1}}}\right) \alpha_{2}+X \sin \sigma\right],
\end{array}\right.
$$

$$
\left\{\begin{array}{l}
\frac{\partial \alpha_{2}}{\partial u}=\frac{\sqrt{E}}{\sin \sigma}\left[\sin (\theta+\omega)\left(\cos \sigma+\sqrt{\frac{\rho}{\rho_{1}}}\right) \alpha_{1}-X \sin \sigma \cos (\theta+\omega)\right], \\
\frac{\partial \alpha_{2}}{\partial v}=\frac{\sqrt{G}}{\sin \sigma}\left[\sin (\theta-\omega)\left(\cos \sigma-\sqrt{\frac{\rho_{p}}{\rho_{1}}}\right) \alpha_{1}-X \sin \sigma \cos (\theta-\omega)\right] .
\end{array}\right.
$$

With the aid of these formulas we show that when the expressions (23) for $X_{I}^{\prime}$ and $X_{2}^{\prime}$ are substituted in (3I) equations (25) must be satisfied and also

$$
\left\{\begin{array}{l}
\frac{\partial \theta_{\mathrm{I}}}{\partial u}-A_{\mathrm{I}}+\sqrt{E} \frac{\sin (\theta+\omega)}{\sin \sigma}\left(\cos \sigma \sqrt{\frac{\rho}{\rho_{\mathrm{I}}}}+\mathrm{I}\right)=0, \\
\frac{\partial \theta_{\mathrm{I}}}{\partial v}+B_{\mathrm{I}}+\sqrt{G} \frac{\sin (\theta-\omega)}{\sin \sigma}\left(\cos \sigma \sqrt{\frac{\rho_{1}}{\rho_{I}}}-\mathrm{I}\right)=0 .
\end{array}\right.
$$

\section{\2.}

\section{Normal $W$-congruences.}

3. We consider now the case $\sigma=\frac{\pi}{2}$. Equations ( 16 ) reduce in this case to

(37)

$$
\begin{aligned}
& \int \frac{\partial \log w_{\mathrm{I}}}{\partial u}=\sqrt{\frac{p_{\mathrm{I}}}{\rho}} \sqrt{E} \cos (\theta+\omega), \\
& \left\langle\frac{\partial \log w_{1}}{\partial v}=-\sqrt{\frac{p_{I}}{\rho}} \sqrt{G} \cos (\theta-\omega),\right. \\
& \left\{\begin{array}{l}
\frac{\partial \log \sqrt{\rho_{p_{I}}}}{\partial u}=\sqrt{E} \cos (\theta+\omega)\left(1 \sqrt{\frac{\rho}{\rho_{1}}}-1 \sqrt{\frac{\rho_{I}}{\rho}}\right), \\
\frac{\partial \log \sqrt{\rho P_{I}}}{\partial v}=-\sqrt{G} \cos (\theta-\omega)\left(\sqrt{\frac{\rho}{\rho_{I}}}-\sqrt{\frac{\rho_{I}}{\rho}}\right),
\end{array}\right. \\
& \int \frac{\partial \theta}{\partial u}-A=-\sqrt{E} \sqrt{\frac{\rho}{\rho_{1}}} \sin (\theta+\omega), \\
& j \frac{\partial \theta}{\partial v}+B=\sqrt{G} \sqrt{\frac{\rho}{\rho_{1}}} \sin (\theta-\omega) \text {. }
\end{aligned}
$$

The condition of integribility of the last equations is satisfied identically, whereas 
the conditions of integribility of $(37)$ and $\left(3^{8}\right)$ require that

$$
\cos (\theta+\omega) \sqrt{E} \frac{\partial \log \sqrt{\rho}}{\partial v}+\cos (\theta-\omega) \sqrt{G} \frac{\partial \log \sqrt{\rho}}{\partial u}=0 .
$$

In order to determine normal $W$-congruences one must express the condition that $\theta$ given by (40) satisfies the preceding equations. It is important to observe, however, that from the form of (40) it follows that a surface satisfying the conditions can be the focal surface of only one normal $W$-congruence.

In consequence of (39) equations (I0) become reducible by (23) to the form

$$
\left\{\begin{array}{l}
\frac{\partial X^{\prime}}{\partial u}=\sqrt{E}\left[\cos (\theta+\omega)\left(-\sin \theta_{1} X_{1}^{\prime}+\cos \theta_{1} X_{2}^{\prime}\right)-\sqrt{\frac{\rho}{\rho_{1}}} \sin (\theta+\omega)\left(\cos \theta_{1} X_{1}^{\prime}+\sin \theta_{1} X_{2}^{\prime}\right)\right] \\
\frac{\partial X^{\prime}}{\partial v}=\sqrt{G}\left[\cos (\theta-\omega)\left(-\sin \theta_{1} X_{1}^{\prime}+\cos \theta_{1} X_{2}^{\prime}\right)+\sqrt{\frac{\rho_{1}}{\rho_{1}}} \sin (\theta-\omega)\left(\cos \theta_{1} X_{1}^{\prime}+\sin \theta_{1} X_{2}^{\prime}\right)\right]
\end{array}\right.
$$

Comparing these with (24), we are brought to the equations

$$
\left\{\begin{array}{l}
\sin \left(\theta_{1}+\omega_{1}\right) \cos (\theta+\omega)+\sqrt{\frac{\rho}{\rho_{1}}} \cos \left(\theta_{1}+\omega_{1}\right) \sin (\theta+\omega)=0, \\
\sin \left(\theta_{1}-\omega_{1}\right) \cos (\theta-\omega)-\sqrt{\frac{\rho}{\rho_{1}}} \cos \left(\theta_{1}-\omega_{1}\right) \sin (\theta-\omega)=0 .
\end{array}\right.
$$

$$
\sqrt{E_{1}}=-\sqrt{\frac{\rho}{\rho_{1}}} \sqrt{E} \frac{\sin (\theta+\omega)}{\sin \left(\theta_{\mathrm{I}}+\omega_{1}\right)}, \quad \sqrt{G_{\mathrm{I}}}=\sqrt{\frac{\rho}{\rho_{1}}} \sqrt{G} \frac{\sin (\theta-\omega)}{\sin \left(\theta_{1}-\omega_{1}\right)} .
$$

In consequence of (42) equations (37) are equivalent to (26) when in the latter we put $\sigma=\frac{\pi}{2}$. Hence all of the formulas (19), (26), (29), (36) are equally true for $\sigma=\frac{\pi}{2}$.

The following known theorems concerning normal $W$-congruences are immediate consequences of equations $(38)$ and $(40)$ :

The total curvature of the focal surfaces at corresponding points is equal only when these surfaces are pseudospherical.

There is a functional relation between the total curvatures of the focal sheets of any $W$-congruence.

\section{$\int 3$.}

Transformations $\Omega$ of conjugate systems with equal tangential invariants.

4. Let $S$ be a surface with the spherical representation of a conjugate parametric system given by (I) satisfying the conditions involved in (2). The surface $\Sigma$ with the spherical representation (I) of its asymptotic lines is the associate of $S$ for the given 
parametric conjugate system. In this sense we shall refer to it as the associate of $S$. The tangential coordinates $X, Y, Z, W$ of $S$ are solutions of (7). The point coordinates $(x, y, z)$ are given by expressions of the form $\left.{ }^{\mathrm{II}}\right)$

(44) $x=W X+\frac{\mathrm{I}}{E G \sin ^{2} 2 \omega}\left[\frac{\partial W}{\partial u}\left(G \frac{\partial X}{\partial u}-F \frac{\partial X}{\partial v}\right)+\frac{\partial W}{\partial v}\left(E \frac{\partial X}{\partial v}-F \frac{\partial X}{\partial u}\right)\right]$, which in consequence of equations (4) may be written

(45) $x=W X+\frac{\mathrm{I}}{\sqrt{\overline{E G} \sin 2 \omega}}\left[\sqrt{G} \frac{\partial W}{\partial u}\left(X_{1} \cos \omega+X_{2} \sin \omega\right)+\sqrt{E} \frac{\partial W}{\partial v}\left(-X_{1} \cos \omega+X_{2} \sin \omega\right)\right]$.

If $D, D^{\prime}=\mathrm{o}, D^{\prime \prime}$ denote the second fundamental coefficients of $S$, we have

$$
\left\{\begin{array}{l}
\frac{\partial x}{\partial u}=\frac{-D}{\sqrt{E} \sin 2 \omega}\left(\cos \omega X_{1}+\sin \omega X_{2}\right), \\
\left.\frac{\partial x}{\partial v}=\frac{D^{\prime \prime}}{\sqrt{G} \sin 2 \omega}\left(\cos \omega X_{1}-\sin \omega X_{2}\right)^{\mathbf{1}}\right) .
\end{array}\right.
$$

Moreover, we have

$$
\left\{\begin{array}{c}
D=-\left[\frac{\partial^{2} W}{\partial u^{2}}-\left\{\begin{array}{c}
\mathrm{II} \\
\mathrm{I}
\end{array}\right\}^{\prime} \frac{\partial W}{\partial u}-\left\{\begin{array}{c}
\mathrm{II} \\
2
\end{array}\right\}^{\prime} \frac{\partial W}{\partial v}+E W\right], \\
\left.D^{\prime \prime}=-\left[\frac{\partial^{2} W}{\partial v^{2}}-\left\{\begin{array}{c}
22 \\
\mathrm{I}
\end{array}\right\}^{\prime} \frac{\partial W}{\partial u}-\left\{\begin{array}{c}
22 \\
2
\end{array}\right\}^{\prime} \frac{\partial W}{\partial v}+G W\right]{ }^{\mathrm{r} 3}\right),
\end{array}\right.
$$

where in consequence of (3) the expressions for CHristoffel symbols may be put in the form

(48)

$$
\left\{\begin{array}{l}
\left\{\begin{array}{c}
\mathrm{I} I \\
\mathrm{I}
\end{array}\right\}^{\prime}=\frac{\partial \log \sqrt{E}}{\partial u}+2 \cot 2 \omega \frac{\partial \omega}{\partial u}-\sqrt{\frac{E}{G}} \cos 2 \omega \frac{\partial \log \sqrt{\rho}}{\partial v}, \\
\left\{\begin{array}{c}
\mathrm{II} \\
2
\end{array}\right\}^{\prime}=\frac{E}{G} \frac{\partial \log \sqrt{\rho}}{\partial v}-2 \sqrt{\frac{E}{G}} \frac{\mathrm{I}}{\sin 2 \omega} \frac{\partial \omega}{\partial u}, \\
\left\{\begin{array}{c}
22 \\
\mathrm{I}
\end{array}\right\}^{\prime}=\frac{G}{E} \frac{\partial \log \sqrt{\rho}}{\partial u}-2 \sqrt{\frac{G}{E}} \frac{\mathrm{I}}{\sin 2 \omega} \frac{\partial \omega}{\partial v}, \\
\left\{\begin{array}{c}
22 \\
2
\end{array}\right\}^{\prime}=\frac{\partial \log \sqrt{G}}{\partial v}+2 \cot 2 \omega \frac{\partial \omega}{\partial v}-\sqrt{\frac{G}{E}} \cos 2 \omega \frac{\partial \log \sqrt{\rho}}{\partial u} .
\end{array}\right.
$$

The function $W_{\mathrm{t}}$ determined by the equations

$$
\left\{\begin{array}{l}
\frac{\partial}{\partial u}\left(\sqrt{\rho \rho_{1}} w_{1} W_{\mathrm{l}}\right)=-\rho\left(w_{\mathrm{r}} \frac{\partial W}{\partial u}-W \frac{\partial w_{\mathrm{r}}}{\partial u}\right), \\
\frac{\partial}{\partial v}\left(\sqrt{\rho \rho_{1}} w_{1} W_{\mathrm{r}}\right)=\rho\left(w_{\mathrm{r}} \frac{\partial W}{\partial v}-W \frac{\partial w_{\mathrm{r}}}{\partial v}\right),
\end{array}\right.
$$

and $X^{\prime}, Y^{\prime}, Z^{\prime}$ are the tangential coordinates of a surface $S_{1}$, of which $\Sigma_{1}$ given by (I2) is the associate.

\footnotetext{
${ }^{11}$ ) Loc. cit. ${ }^{6}$ ), p. 163 .

${ }^{12}$ ) Loc. cit. ${ }^{6}$ ), p. 200.

13) Loc. cit. ${ }^{6}$ ), p. 164.
} 
The coordinates $\left(x_{1}, y_{1}, z_{1}\right)$ of $S_{1}$ are of the form

(5o)

$$
\begin{aligned}
& \left\{x_{\mathrm{t}}=W_{\mathrm{I}} X^{\prime}+\frac{\mathrm{I}}{\sqrt{E_{1} G_{\mathrm{I}}} \sin 2 \omega_{\mathrm{I}}}\left[\sqrt{G_{\mathrm{t}}} \frac{\partial W_{\mathrm{I}}}{\partial v}\left(X_{\mathrm{t}}^{\prime} \cos \omega_{\mathrm{I}}+X_{2}^{\prime} \sin \omega_{1}\right)\right.\right. \\
& \left.+\sqrt{E_{1}} \frac{\partial W_{1}}{\partial v}\left(-X_{1}^{\prime} \cos \omega_{1}+X_{2}^{\prime} \sin \omega_{1}\right)\right] \text {. }
\end{aligned}
$$

We substitute in (5o) the expressions for $\frac{\partial W_{1}}{\partial u}$ and $\frac{\partial W_{1}}{\partial v}$ given by (49); also the expressions for $X^{\prime}, X_{1}^{\prime}, X_{2}^{\prime}$ given by (9) and (23). If we put

$$
R=\frac{\rho}{P_{\mathrm{I}}} \frac{\sqrt{\overline{E G}}}{\sqrt{\bar{E}_{\mathrm{I}} G_{\mathrm{I}} \sin 2 \omega_{\mathrm{I}}}}\left[\frac{W \cos \sigma-W_{\mathrm{I}}}{\sin \sigma}+\frac{\mathrm{I}}{\sqrt{E}} \frac{\sin (\theta-\omega)}{\sin 2 \omega} \frac{\partial W}{\partial u}-\frac{\mathrm{I}}{\sqrt{\bar{G}}} \frac{\sin (\theta+\omega)}{\sin 2 \omega} \frac{\partial W}{\partial v}\right],
$$

the result of the above substitution becomes

$$
\left\{\begin{aligned}
\frac{x_{1}-x}{R} & =X_{1}\left\{\cos \omega\left[\cot \left(\theta_{1}-\omega_{1}\right) \sin (\theta-\omega)-\cot \left(\theta_{1}+\omega_{1}\right) \sin (\theta+\omega)\right]-\cos \sigma \sin \theta \sin 2 \omega\right\} \\
& +X_{2}\left\{-\sin \omega\left[\cot \left(\theta_{1}-\omega_{1}\right) \sin (\theta-\omega)+\cot \left(\theta_{\mathrm{r}}+\omega_{1}\right) \sin (\theta+\omega)\right]+\cos \sigma \cos \theta \sin 2 \omega\right\} \\
& +X \sin \sigma \sin 2 \omega .
\end{aligned}\right.
$$

Since $W$, enters only in the factor $R$ and not in the right-hand member of the equation, and since $W_{1}$ is determined only to within the additive function $\frac{c}{\sqrt{P p_{1}} w_{1}}$, where $c$ is an arbitrary constant, we see that on the line joining corresponding points of $S$ and $S_{1}$ there is an infinity of points describing surfaces with the same spherical representation of a conjugate system as $S_{1}$. In like manner in equations (49) $W$ may be replaced by $W+c w_{1}$ without changing the right-hand member. Hence on the line referred to there are an infinity of points describing conjugate systems parallel to the conjugate system on $S$. One knows that if two conjugate systems are parallel the lines joining corresponding points form a congruence whose developables cut the two surfaces in these conjugate lines. In view of these results we have the theorem:

If $S$ is a surface referred to a conjugate system with equal tangential invariants and $\mathbf{\Sigma}$ its associate surface; if $\mathbf{\Sigma}_{1}$ is a surface which with $\mathbf{\Sigma}$ constitute the focal sheets of a $W$-congruence, determined by a set of functions $\theta_{,} \theta_{1}, w_{\mathrm{I}}, \sigma$; when one draws through points of $S$ lines baving the direction determined by the right-hand member of (52), these lines generate a congruence whose developables meet $S$ in the parametric conjugate system, and one can determine upon these lines by one quadrature an infinity of points each generating a surface $S_{1}$, cut in a conjugate system by the developlables of the congruence, and $\Sigma_{1}$ is the associate surface of $S_{1}$.

We say that $S$ and $S_{1}$ are in the relation of a transformation $\mathbf{\Omega}$.

We shall show that for one of the surfaces associate to a given $\boldsymbol{\Sigma}$ and for a given set of functions $\theta, \theta_{\mathrm{I}}, w_{\mathrm{I}}, \sigma$ all the lines of the congruence pass through the origin. The analytical condition for this is that there exist a function $t$ such that

$$
x_{\mathrm{r}}-x=t x, \quad y_{\mathrm{r}}-y=t y^{\prime}, \quad z_{1}-z=t z .
$$


From (44) and (52) it follows that we must have

(53)

$$
\left\{\begin{array}{c}
R \cos \sigma \sin 2 \omega=t V \\
=\frac{t}{\sqrt{E G} \sin 2 \omega}\left(\sqrt{G} \frac{\partial W}{\partial u}-\sqrt{E} \frac{\partial W}{\partial v}\right), \\
R\left\{\cot \left(\theta_{1}-\omega_{1}\right) \sin (\theta-\omega)+\cot \left(\theta_{1}+\omega_{1}\right) \sin (\theta+\omega)-2 \cos \sigma \cos \theta \cos \omega\right\} \\
=\frac{-t}{\sqrt{E G} \sin 2 \omega}\left(\sqrt{G} \frac{\partial W}{\partial u}+\sqrt{E} \frac{\partial W}{\partial v}\right) .
\end{array}\right.
$$

One finds readily that these equations are consistent when $W=w_{1}$, given by (26), and only in this case.

Referring to (49), we see that the functions $W_{\mathrm{I}}$ are given by

$$
W_{\mathrm{t}}=\frac{c}{\sqrt{\rho p_{\mathrm{I}}} w_{\mathrm{I}}}
$$

We denote by $\bar{S}$ and $\bar{S}_{1}$ the surfaces associate to $\Sigma$ and $\Sigma_{1}$ respectively, for which $W=w_{1}$ and equation (54) holds.

The surface $\bar{S}$ determines an infinitesimal deformation of $\Sigma$ for which the coordinates of the surface $\Sigma^{\prime}$ corresponding to $\mathbf{\Sigma}$ with orthogonality of linear elements are equal to

$$
\left.\sqrt{\rho p_{1}} w_{1} X^{\prime}, \quad \sqrt{\rho p_{1}} w_{1} Y^{\prime}, \quad \sqrt{\rho p_{1}} w_{1} Z^{\prime} \quad{ }^{14}\right)
$$

Owing to the reciprocil relation of $\mathbf{\Sigma}$ and $\mathbf{\Sigma}$ the latter admits an infinitesimal deformation for which $\Sigma$ is the surface corresponding with orthogonality of linear elements. The coordinates of the corresponding associate surface $\Sigma_{\mathrm{i}}^{\prime}$ are $\frac{X}{w_{\mathrm{I}}}, \frac{Y}{w_{\mathrm{I}}^{\prime}}, \frac{Z}{w_{\mathrm{i}}}$. If we consider $\Sigma^{\prime}$ as the associate surface in an infinitesimal deformation of $\Sigma_{I}^{\prime}$ the surface corresponding to the latter with orthogonality of linear elements is $\Sigma_{1}$ given by (I2) ${ }^{15}$ ). Moreover, $\bar{S}_{1}$ is the associate of $\Sigma_{1}$ corresponding to $\Sigma_{1}^{\prime}$. Hence $\Sigma, \Sigma^{\prime}, \Sigma_{1}^{\prime}, \Sigma_{1}, \bar{S}$ and $\bar{S}_{\mathrm{I}}$ form part of a group of the so-called twelve surfaces of Darboux ${ }^{\mathrm{I}}$ ).

\section{$\int 4$.}

\section{Theorem of permutability for the transformations $\Omega$.}

5. Suppose that we have two solutions $w_{1}$ and $w_{2}$ of equation (7). The function $w_{2}$ leads to a $W$-congruence of which $\Sigma$ and a surface $\Sigma_{2}$ are the focal sheets; we denote by $p_{2}$ the function for $\Sigma_{2}$ analogous to $p$ for $\Sigma$.

14) Loc. cit. ${ }^{6}$ ), p. 420.

15) Loc cit. 6), p. 419.

16) Cfr. G. DARBoux, Lesons sur la théorie générale des surfaces et les applications géométriques du Calcul infinitésimal (Paris, Gauthier-Villars), Vol. IV (1896), pp. 48.72; also: L. P. EIsenharT, The Twelve Surfaces of DARBOUX and the Transformation of MOUTARD [American Journal of Mathematics, Vol. XXXII (I910), pp. I7-36]. 
By the transformation of Moutard two functions $w_{x}^{\prime}$ and $w_{2}^{\prime}$ are given by the quadratures

$$
\left\{\begin{array}{l}
\frac{\partial}{\partial u}\left(\sqrt{\rho \rho_{i}} w_{i} w_{i}^{\prime}\right)=-\rho\left(w_{i} \frac{\partial w_{i}}{\partial u}-w_{j} \frac{\partial w_{i}}{\partial u}\right), \\
\left.\frac{\partial}{\partial v}\left(\sqrt{\rho \rho_{i}} w_{i} w_{i}^{\prime}\right)=\rho\left(w_{i} \frac{\partial w_{j}}{\partial v}-w_{i} \frac{\partial w_{i}^{\prime}}{\partial v}\right)^{{ }^{s} 7}\right)
\end{array}\right.
$$

The functions $w_{i}^{\prime}$ are determined only to within the additive quantity $\frac{c}{\sqrt{\rho p_{i}} w_{i}}$, where $c$ is an arbitrary constant. These constants can be chosen so that pairs of functions satisfy the relation

$$
\sqrt{\rho_{\rho} \rho_{1}} w_{1} w_{1}^{\prime}+\sqrt{\rho \rho_{2}} w_{2} w_{2}^{\prime}=0 .
$$

With this pairing of the functions $w_{1}^{\prime}$ and $w_{2}^{\prime}$ we have

$$
\sqrt{\rho_{1}} w_{1}^{\prime} \frac{\partial^{2}}{\partial u \partial v}\left(\frac{\mathrm{I}}{\sqrt{\rho_{1}} w_{1}^{\prime}}\right)=\sqrt{\rho_{2}} w_{2}^{\prime} \frac{\partial^{2}}{\partial u \partial v}\left(\frac{1}{\sqrt{\rho_{2}} w_{2}^{\prime}}\right) \text {. }
$$

Also one has that $w_{1}^{\prime}$ and $w_{2}^{\prime}$ are solutions of

$$
\frac{\partial^{2}}{\partial u \partial v}\left(\sqrt{\rho_{i}} \theta_{i}\right)=\sqrt{\rho} w_{i} \frac{\partial^{2}}{\partial u \partial v}\left(\frac{1}{\sqrt{\rho} w_{i}}\right) \cdot \sqrt{\rho_{i}} \theta_{i} \quad(i=1,2) .
$$

If we compare (55) with (49) we note that $w_{1}^{\prime}$ is obtained from $w_{2}$ just as $W_{1}$ is obtained from $W$. Hence $w_{1}^{\prime}$ and $W$, are solutions of the same equation, namely (58) for $i=\mathrm{I}$. Accordingly $2 v_{\mathrm{r}}^{\prime}$ can be used to transform $W_{\mathrm{i}}$ into a function $W_{\mathrm{1}}^{\prime}$. If we denote by $W_{2}$ the function obtained from $W$ with the aid of $w_{2}$ by means of equations similar to (49), it follows that $w_{2}^{\prime}$ and $W_{2}$ satisfy the same equation, namely (58), for $i=2$. Hence $w_{2}^{\prime}$ enables us to obtain from $W_{2}$ a function $W_{2}^{\prime}$ by equations of the form (49). It is our purpose to show that in consequence of $(56)$ the functions $W_{1}^{\prime}$ and $W_{2}^{\prime}$ are equal to one another; we denote them by $W^{\prime}$.

In this case we must have

$$
\left\{\begin{array}{l}
\frac{\partial}{\partial u}\left(\sqrt{\rho^{\prime} \rho_{i}} w_{i}^{\prime} W^{\prime}\right)=-\rho_{i}\left(w_{i}^{\prime} \frac{\partial W_{i}}{\partial u}-W_{i} \frac{\partial w_{i}^{\prime}}{\partial u}\right), \\
\frac{\partial}{\partial v}\left(\sqrt{\rho^{\prime} \rho_{i}} w_{i}^{\prime} W^{\prime}\right)=\rho_{i}\left(w_{i}^{\prime} \frac{\partial W_{i}}{\partial v}-W_{i} \frac{\partial w_{i}^{\prime}}{\partial v}\right),
\end{array} \quad(i=1,2)\right.
$$

where $\rho^{\prime}$ is a function analogous to $p$, and also

$$
\left\{\begin{array}{l}
\frac{\partial}{\partial u}\left(\sqrt{\rho \rho_{i}} w_{i} W_{i}\right)=-\rho\left(w_{i} \frac{\partial W}{\partial u}-W \frac{\partial w_{i}}{\partial u}\right), \\
\frac{\partial}{\partial v}\left(\sqrt{\rho \rho_{i}} w_{i} W_{i}\right)=\rho\left(w_{i} \frac{\partial W}{\partial v}-W \frac{\partial w_{i}}{\partial v}\right),
\end{array}\right.
$$

these equations being similar to (49).

I 7) L. Bianchi, loc. cit. 3), p. 70. 
If we take the first of equations (59) for $i=\mathrm{I}$ and 2 and substitute for $\frac{\partial W_{i}}{\partial u}$ from (6o), and then eliminate $\frac{\partial W^{\prime}}{\partial u}$, we obtain

$$
\sqrt{\rho^{\prime}} W^{\prime}=\sqrt{\rho} W+t\left(W_{1} \sqrt{\rho_{1}}-W_{2} \sqrt{\rho_{2}}\right)
$$

where

$$
t=\frac{w_{1} \sqrt{p}}{w_{2}^{\prime} \sqrt{\rho_{2}}}=-\frac{w_{2} \sqrt{p}}{w_{1}^{\prime} \sqrt{\rho_{1}}}
$$

If we proceed in like manner with the second of equations (59) for $i=\mathrm{I}$ and 2 , we are brought to the same result.

Furthermore, one shows readily that the expression (6I) satisfies equations (59), so that the assumption that $W_{1}^{\prime}$ and $W_{2}^{\prime}$ are equal is correct.

The direction-cosines of the normal to $S_{1}$ are obtained from those of $S$ by equations (II) which are of the same form as (6o). In like manner the direction-cosines $X^{\prime \prime}, Y^{\prime \prime}, Z^{\prime \prime}$ of the normal to $S_{2}$ are obtained from those of $S$ by $(60)$. Evidently the direction-cosines $\bar{X}, \bar{Y}, \bar{Z}$ of the normal to $S^{\prime}$ for which $W^{\prime}$ given by (6I) is a tangential coordinate are given by expressions of the form

$$
\sqrt{\rho^{\prime}} \bar{X}=\sqrt{\rho} X+t\left(X^{\prime} \sqrt{\rho_{1}}-X^{\prime \prime} \sqrt{\rho_{2}}\right) \text {. }
$$

From (61) and (63) it follows that the tangent planes of $S, S_{1}, S_{2}, S^{\prime}$ at corresponding points meet in a point.

If $\Sigma, \Sigma_{1}, \Sigma_{2}$ are the surfaces associate to $S, S_{1}, S_{2}$, so that $\Sigma$ and $\Sigma_{1}$ are the focal sheets of a $W$-congruence, and also $\Sigma$ and $\Sigma_{2}$, the associate $\Sigma^{\prime}$ of $S^{\prime}$ can be so placed in space that $\Sigma_{1}$ and $\Sigma^{\prime}$ are the focal sheets of a $W$-congruence and likewise $\Sigma_{2}$ and $\Sigma^{\prime}$ as Biancir has shown. In fact, the cartesian coordinates of $\Sigma^{\prime}$ are of the form

$$
\xi^{\prime}=\xi+t \sqrt{\rho_{1} p_{2}}\left(Y^{\prime} Z^{\prime \prime}-Z^{\prime} Y^{\prime \prime}\right) \text {. }
$$

It was this result of BIANCHI which gave a geometrical interpretation of the theorem of Moutard. Our results have given another interpretation. There are other properties of the theorem of permutability which we shall develop now.

6. We denote by $s_{1}$ and $s_{2}$ the surfaces whose parametric conjugate system has the spherical representation (I), and which are determined by the respective functions $w_{1}$ and $w_{2}$. We denote by $x_{01}, y_{01}, z_{01} ; x_{02}, y_{02}, z_{02}$ the coordinates of $s_{1}$ and $s_{2}$. Then for $i=\mathrm{I}$ and 2

$$
x_{\mathrm{o} i}=w_{i} X+\frac{\mathrm{I}}{\sqrt{E G} \sin 2 \omega}\left[\left(\sqrt{G} \frac{\partial w_{i}}{\partial u}-\sqrt{E} \frac{\partial w_{i}}{\partial v}\right) \cos \omega X_{\mathrm{s}}+\left(\sqrt{G} \frac{\partial w_{i}}{\partial u}+\sqrt{E} \frac{\partial w_{i}}{\partial v}\right) \sin \omega X_{2}\right] .
$$

It is readily shown that equation (52) can be written

$$
x_{i}-x=\frac{R_{i} \sin \sigma_{i} \sin 2 \omega}{w_{i}} x_{o i} .
$$$$
(i=\mathrm{I}, 2) \text {. }
$$

If we compare equations ( $5 S$ ) with (49), we observe that the surface $s_{1}^{\prime}$ determined 
by $w_{1}^{\prime}$ is a transform of $s_{2}$. Hence its coordinates $x_{\mathrm{or}}^{\prime}, y_{\mathrm{O}}^{\prime}, z_{\mathrm{or}}^{\prime}$ are given by expressions of the form

$$
x_{01}^{\prime}-x_{02}=\frac{R_{O 1}^{\prime} \sin \sigma_{I} \sin 2 \omega}{w_{1}} x_{o 1},
$$

where

$$
R_{\mathrm{o}}^{\prime}=\frac{\rho}{\rho_{1}} \frac{\sqrt{E G}}{\sqrt{E_{1} G_{1} \sin 2 \omega_{1}}}\left[\frac{w_{2} \cos \sigma_{1}-w_{1}^{\prime}}{\sin \sigma_{1}}+\frac{\mathrm{I}}{\sqrt{E}} \frac{\sin (\theta-\omega)}{\sin 2 \omega} \frac{\partial w_{2}}{\partial u}-\frac{\mathrm{I}}{\sqrt{\bar{G}}} \frac{\sin (\theta+\omega)}{\sin 2 \omega} \frac{\partial \omega_{2}}{\partial v}\right]
$$

Since $w_{\mathrm{r}}^{\prime}$ is the transforming function of $S_{1}$, it follows from equations analogous to (66) that

$$
x^{\prime}-x_{1}=\frac{R_{\mathrm{t}}^{\prime} \sin \sigma_{1}^{\prime} \sin 2 \omega_{\mathrm{I}}}{w_{1}^{\prime}} x_{\mathrm{ol}}^{\prime},
$$

where, analogously to (68),

$$
\begin{aligned}
& \left\{R_{\mathrm{I}}^{\prime}=\frac{\rho_{\mathrm{I}}}{\rho^{\prime}} \frac{\sqrt{E_{\mathrm{I}} \bar{G}_{\mathrm{I}}}}{\sqrt{E^{\prime} G^{\prime} \sin 2 \omega^{\prime}}}\left[\frac{W_{\mathrm{I}} \cos \sigma_{\mathrm{I}}^{\prime}-W^{\prime}}{\sin \sigma_{\mathrm{I}}^{\prime}}\right.\right. \\
& \left.+\frac{\mathrm{I}}{\sqrt{E_{1}}} \frac{\sin \left(\theta^{\prime}-\omega_{1}\right)}{\sin 2 \omega_{1}} \frac{\partial W_{1}}{\partial u}-\frac{\mathrm{I}}{\sqrt{G_{1}}} \frac{\sin \left(\theta^{\prime}+\omega_{1}\right)}{\sin 2 \omega_{1}} \frac{\partial W_{1}}{\partial v}\right] .
\end{aligned}
$$

From (66), (67) and (69) it follows that the point $M^{\prime}$ of $S^{\prime}$ with coordinates $x^{\prime}, y^{\prime}, z^{\prime}$ lies in the plane determined by $M, M_{1}, M_{2}$. In fact $M^{\prime}$ is the point of intersection of the lines through $M_{1}$ and $M_{2}$ whose direction parameters are proportional to $x_{01}^{\prime}, y_{01}^{\prime}, z_{01}^{\prime} ; x_{02}^{\prime}, y_{02}^{\prime}, z_{02}^{\prime}$ respectively.

We have observed that $w_{1}^{\prime}$ is determined only to within the additive function $\frac{c}{\sqrt{\rho \rho_{1}} w_{1}}$. Accordingly we put

$$
w_{1}^{\prime}=\bar{w}_{\mathrm{r}}^{\prime}+\frac{c}{\sqrt{\bar{\rho}_{1} w_{\mathrm{r}}}}
$$

where $\bar{w}_{t}^{\prime}$ is independent of $c$. In order that $(56)$ may hold $w_{2}^{\prime}$ must be of the form

$$
w_{2}^{\prime}=\bar{w}_{2}^{\prime}-\frac{c}{\sqrt{\rho \rho_{2}} w_{2}^{\prime}},
$$

it being understood that $\bar{w}_{\mathrm{x}}^{\prime}$ and $\bar{w}_{2}^{\prime}$ satisfy $(56)$. From (67) we have accordingly

$$
x_{01}^{\prime}=x_{02}+\left(P_{1}+c Q_{1}\right) x_{01}, \quad x_{02}^{\prime}=x_{01}+\left(P_{2}+c Q_{2}\right) x_{02},
$$

where $P_{1}, Q_{1}, P_{2}, Q_{2}$ are independent of $c$.

Evidently the two pencils of lines through $M_{1}$ and $M_{2}$ whose direction-parameters have the values (73) respectively are projective. Hence the points $M^{\prime}$ lie on a conic. From (7I) it follows that as $c$ becomes very large the term $\frac{c}{\sqrt{\rho \rho_{\mathrm{I}}} w_{\mathrm{I}}}$ is the controlling term. Hence for $c=\infty$ we have the point $M$.

The question arises as to whether the conic is degenerate so that in fact the pencils are perspective and all the points lie on a line. In order to settle this question we determine the coordinates of $M^{\prime}$. 
From (6r) and (63) we have that $S^{\prime}$ is the envelope of the plane (74) $\sum x^{\prime}\left[(T+c) X+w_{1} w_{2}\left(X^{\prime} \sqrt{\rho \rho_{1}}-X^{\prime \prime} \sqrt{\rho_{\rho_{2}}}\right)\right]=(T+c) W+w_{1} w_{2}\left(W_{1} \sqrt{\rho \rho_{1}}-W_{2} \sqrt{\rho \rho_{2}}\right)$, where

$$
T=w_{2} w_{2}^{\prime} \sqrt{\rho \rho_{2}} .
$$

If we differentiate this equation with respect to $u$ and $v$ respectively, and in the reduction make use of the preceding results, we have

(75)

$$
\left\{\begin{array}{l}
\sum x^{\prime}\left[(T+c) \frac{\partial X}{\partial u}+w_{1} \frac{\partial w_{2}}{\partial u} X^{\prime} \sqrt{\rho \rho_{1}}-w_{2} \frac{\partial w_{1}}{\partial u} X^{\prime \prime} \sqrt{\rho \rho_{2}}\right] \\
=(T+c) \frac{\partial W}{\partial u}+w_{1} \frac{\partial w_{2}}{\partial u} W_{1} \sqrt{\rho \rho_{1}}-w_{2} \frac{\partial w_{1}}{\partial u} W_{2} \sqrt{\rho \rho_{2}}, \\
\sum x^{\prime}\left[(T+c) \frac{\partial X}{\partial v}+w_{1} \frac{\partial w_{2}}{\partial v} X^{\prime} \sqrt{\rho \rho_{1}}-w_{2} \frac{\partial w_{1}}{\partial v} X^{\prime \prime} \sqrt{\rho \rho_{2}}\right] \\
=(T+c) \frac{\partial W}{\partial v}+w_{1} \frac{\partial w_{2}}{\partial v} W_{1} \sqrt{\rho \rho_{1}}-w_{2} \frac{\partial w_{1}}{\partial v} W_{2} \sqrt{\rho \rho_{2}} .
\end{array}\right.
$$

If for the sake of brevity we put

$$
(\theta, \varphi, \psi)=\left|\begin{array}{ccc}
\theta & \varphi & \psi \\
\frac{\partial \theta}{\partial u} & \frac{\partial \varphi}{\partial u} & \frac{\partial \psi}{\partial u} \\
\frac{\partial \theta}{\partial v} & \frac{\partial \varphi}{\partial v} & \frac{\partial \psi}{\partial v}
\end{array}\right|, \quad H=\sqrt{E G} \sin 2 \omega
$$

the determinant $\Delta$ of equations $(74)$ and (75) is reducible to

$$
\begin{aligned}
\Delta=(T+c)^{3} H & +(T+c)^{2} H\left[w_{1} \sqrt{\rho \rho_{1}} \sum X^{\prime} x_{02}-w_{2} \sqrt{\rho \rho_{2}} \sum X^{\prime \prime} x_{01}\right] \\
& +(T+c) \rho \sqrt{\rho_{1} \rho_{2}} w_{1} w_{2} \sum\left(X^{\prime} Y^{\prime \prime}-X^{\prime \prime} Y^{\prime}\right) \cdot\left(w_{1}, w_{2}, Z\right) .
\end{aligned}
$$

Solving equations (74) and (75) for $x^{\prime}$, we obtain

$$
\left(7^{\prime}\right)\left\{\begin{array}{c}
\Delta x^{\prime}=(T+c)^{3} H x+(T+c)^{2}\left\{\left(W, Y, w_{2}\right) w_{1} \sqrt{\rho \rho_{1}} Z^{\prime}-\left(W, Y, w_{1}\right) w_{2} \sqrt{\rho \rho_{2}} Z^{\prime \prime}\right. \\
-\left(W, Z, w_{2}\right) w_{1} \sqrt{\rho \rho_{1}} Y^{\prime}+\left(W, Z, w_{1}\right) w_{2} \sqrt{\rho \rho_{1}} Y^{\prime \prime}+W_{2} w_{1} \sqrt{\rho_{1} \rho}\left(w_{2}, Y, Z\right) \\
\left.-W_{2} w_{2} \sqrt{\rho \rho_{2}}\left(w_{1}, Y, Z\right)\right\}+(T+c) w_{1} w_{2} \rho \sqrt{\rho_{1} \rho_{2}}\left(\left(W, w_{1}, w_{2}\right)\left(Y^{\prime} Z^{\prime \prime}-Y^{\prime \prime} Z^{\prime}\right)\right. \\
\left.\quad+\left(Y, w_{1}, w_{2}\right)\left(Z^{\prime} W_{2}-Z^{\prime \prime} W_{1}\right)+\left(Z, w_{1}, w_{2}\right)\left(W_{1} Y^{\prime \prime}-W_{2} Y^{\prime}\right)\right\} .
\end{array}\right.
$$

From expressions $(76)$ and $\left(7^{\prime}\right)$ it follows that

$$
\begin{aligned}
& \left(x^{\prime}-x\right) \Delta=a_{\mathrm{1}}(T+c)^{2}+a_{\mathrm{o}}(T+c), \\
& \left(y^{\prime}-y\right) \Delta=b_{\mathrm{1}}(T+c)^{2}+b_{0}(T+c), \\
& \left(z^{\prime}-z\right) \Delta=c_{1}(T+c)^{2}+c_{0}(T+c),
\end{aligned}
$$

where $a_{1}, b_{1}, c_{1} ; a_{0}, b_{0}, c_{0}$ are determinate functions of $u$ and $v$. If the locus of $M^{\prime}$ is to be a straight line, the ratios

$$
\frac{x^{\prime}-x}{y^{\prime}-y}, \quad \frac{x^{\prime}-x}{z^{\prime}-z}
$$


must be independent of $c$. The necessary and sufficient condition for this is that

$$
\frac{a_{1}}{a_{0}}=\frac{b_{1}}{b_{0}}=\frac{c_{1}}{c_{0}} \text {. }
$$

This gives two equations linear in $W_{1}$ and $W_{2}$. The coefficient of $W_{1}$ in these two equations involve $X^{\prime \prime}, Y^{\prime \prime}, Z^{\prime \prime}, w_{1}, w_{2}$. Evidently they cannot be zero for $X^{\prime \prime}$ is independent of $w_{1}$. Hence $W_{1}$ and $W_{2}$ could be found by solving two linear equations. But they are determined by $(60)$ only to within additive functions $\frac{\alpha}{\sqrt{\rho_{1} p_{1}}}$ and $\frac{\beta}{\sqrt{\rho \rho_{2}} w_{2}}$ where $\alpha$ and $\beta$ are constants. Hence in general the above conditions are not satisfied.

Accordingly the theorem of permutability may be stated as follows:

Let $s_{1}$ and $s_{2}$ be two surfaces corresponding to the given surface $S$ with parallelism of tangent planes, and let the tangential coordinates of $s_{1}$ and $s_{2}$ be $X, Y, Z, w_{1}$ and $X, Y, Z, w_{2} ; b_{y}$ means of $w_{1}$ and $w_{2}$ we transform $S$ into two surfaces $S_{1}$ and $S_{2}$; also by means of $w_{1}$ we transform $s_{2}$ into $s_{1}^{\prime}$ and by $w_{2}$ we transform $s_{1}$ into $s_{2}^{\prime}$, the corresponding tangential coordinates of $s_{1}^{\prime}$ and $s_{2}^{\prime}$ being denoted by $w_{1}^{\prime}$ and $w_{2}^{\prime}$.

If through a point $M_{1}$ of $S_{1}$ we draw a line parallel to the radius vector of $s_{1}^{\prime}$ at the corresponding point, and in like manner through the corresponding point $M_{2}$ of $S_{2}$ a line parallel to the radius vector of $s_{2}^{\prime}$ the lines meet in a point $M^{\prime}$ of the plane $M$, $M_{1}, M_{2}$. The locus of $M^{\prime}$, a surface $S^{\prime}$, is the transform of $S_{1}$ by $w_{1}^{\prime}$ and of $S_{2}$ by $w_{2}^{\prime}$. When the parameter in $w_{1}^{\prime}$ varies we get $\infty^{\prime}$ of points $M^{\prime}$ which lie on a conic passing through $M, M_{1}, M_{2}$.

\section{$\$ 5$.}

\section{The Adjoint Congruence.}

7. Since the joins of corresponding points of $S$ and $S_{1}$ form a congruence whose developables cut these surfaces in a conjugate system, the developables of the congruence of lines of intersection of the tangent planes to $S$ and $S_{J}$ correspond to the developables of the former congruence. We say that the new congruence is adjoint to the other. We know that its focal points are the points of intersection of the tangents to the parametric curves of $S$ and $S_{\mathrm{I}}$ at corresponding points. It is our purpose now to find the coordinates $a_{1}, b_{1}, c_{1} ; a_{2}, b_{2}, c_{2}$ of these focal points.

In consequence of $(46)$ and analogous equations for $S_{1}$ it follows that the expressions for $a_{1}$ and $a_{2}$ may be given the form

$$
\left\{\begin{array}{l}
a_{1}=x+\lambda_{1}\left(\cos \omega X_{1}+\sin \omega X_{2}\right)=x_{1}+\mu_{1}\left(\cos \omega_{1} X_{1}^{\prime}+\sin \omega_{1} X_{2}^{\prime}\right), \\
a_{2}=x+\lambda_{2}\left(\cos \omega X_{1}+\sin \omega X_{2}\right)=x_{1}+\mu_{2}\left(\cos \omega_{1} X_{1}^{\prime}-\sin \omega_{1} X_{2}^{\prime}\right),
\end{array}\right.
$$

where $\lambda_{1}, \mu_{1}, \lambda_{2}, \mu_{2}$ are to be determined. 
The equality of the two expressions for $a_{1}$ necessitates the equations

$$
R \sin 2 \omega-\mu_{1} \sin \left(\theta_{1}-\omega_{1}\right)=0,
$$

$R\left\{\cos \omega\left[\cot \left(\theta_{1}-\omega_{1}\right) \sin (\theta-\omega)-\cot \left(\theta_{1}+\omega_{1}\right) \sin (\theta+\omega)\right]-\cos \sigma \sin \theta \sin 2 \omega\right\}$ $+\mu_{1}\left\{\cos \left(\theta_{1}-\omega_{1}\right) \cos \theta+\sin \left(\theta_{1}-\omega_{1}\right) \cos \sigma \sin \theta\right\}-\lambda_{1} \cos \omega=0$,

$R\left\{-\sin \omega\left[\cot \left(\theta_{1}-\omega_{1}\right) \sin (\theta-\omega)+\cot \left(\theta_{1}+\omega_{1}\right) \sin (\theta+\omega)\right]+\cos \sigma \cos \theta \sin 2 \omega\right\}$ $+\mu_{I}\left\{\cos \left(\theta_{I}-\omega_{I}\right) \sin \theta-\sin \left(\theta_{I}-\omega_{I}\right) \cos \sigma \cos \theta\right\}-\lambda_{I} \sin \omega=0$.

From these we have

$$
\lambda_{1}=\frac{R \sin (\theta+\omega) \sin 2 \omega_{1}}{\sin \left(\theta_{1}-\omega_{1}\right) \sin \left(\theta_{1}+\omega_{1}\right)}, \quad \mu_{1}=\frac{R \sin 2 \omega}{\sin \left(\theta_{1}-\omega_{1}\right)} .
$$

In like manner we find

If we put

$$
\lambda_{2}=\frac{R \sin (\theta-\omega) \sin 2 \omega_{1}}{\sin \left(\theta_{1}-\omega_{1}\right) \sin \left(\theta_{1}+\omega_{1}\right)}, \quad \mu_{2}=\frac{R \sin 2 \omega}{\sin \left(\theta_{1}+\omega_{1}\right)} .
$$

(8o) $\quad T=\frac{W_{\mathrm{t}}-W \cos \sigma}{\sin \sigma}-\frac{\mathrm{I}}{\sqrt{E}} \frac{\sin (\theta-\omega)}{\sin 2 \omega} \frac{\partial W}{\partial u}+\frac{\mathrm{I}}{\sqrt{G}} \frac{\sin (\theta+\omega)}{\sin 2 \omega} \frac{\partial W}{\partial v}$, it follows from $(78)$ and (79), when the expression ( 51$)$ for $R$ is substituted, that

$$
\lambda_{\mathrm{r}}=\frac{T}{\sin (\theta-\omega)}, \quad \lambda_{2}=\frac{T}{\sin (\theta+\omega)} .
$$

From (80) we have

$$
\begin{aligned}
& \frac{\partial T}{\partial u}=-\frac{\cos (\theta+\omega)\left(\cos \sigma+\sqrt{\frac{\rho}{P_{1}}}\right) \sqrt{E}}{\sin \sigma} T+\frac{D}{\sqrt{E}} \frac{\sin (\theta-\omega)}{\sin 2 \omega}, \\
& \frac{\partial T}{\partial v}=-\frac{\cos (\theta-\omega)\left(\cos \sigma-\sqrt{\frac{\rho}{P_{1}}}\right) \sqrt{G}}{\sin \sigma} T-\frac{D^{\prime \prime}}{\sqrt{G}} \frac{\sin (\theta+\omega)}{\sin 2 \omega} .
\end{aligned}
$$

Making use of these results, we obtain from (77) by differentiation

$$
\left\{\begin{aligned}
\frac{\partial a_{1}}{\partial u}=\frac{\lambda_{1} \sqrt{E} \sin 2 \omega}{\sin (\theta-\omega)}[-\sin (\theta-\omega) X & +\frac{\cos \sigma+\sqrt{\frac{\rho}{\rho_{1}}}}{\sin \sigma}\left(\cos \omega X_{1}+\sin \omega X_{2}\right) \\
& \left.+\frac{1}{\sqrt{G}} \frac{\partial \log \sqrt{\rho}}{\partial v}\left(\cos \theta X_{1}+\sin \theta X_{2}\right)\right] \\
\frac{\partial a_{1}}{\partial v}= & {\left[\lambda_{1}\left(\frac{\partial \omega}{\partial v}+B\right)-\frac{D^{\prime \prime}}{\sqrt{G}}\right] \frac{\cos \theta X_{1}+\sin \theta X_{2}}{\sin (\theta-\omega)}, }
\end{aligned}\right.
$$


(83)

$$
\left\{\begin{array}{r}
\frac{\partial a_{2}}{\partial u}=-\left[\lambda_{2}\left(\frac{\partial \omega}{\partial u}+A\right)+\frac{D}{\sqrt{E}}\right] \frac{\cos \theta X_{1}+\sin \theta X_{2}}{\sin (\theta+\omega)}, \\
\frac{\partial a_{2}}{\partial v}=\frac{\lambda_{2} \sqrt{G} \sin 2 \omega}{\sin (\theta+\omega)}\left[\sin (\theta+\omega) X-\frac{\cos \sigma-\sqrt{\frac{\rho}{\rho_{1}}}}{\sin \sigma}\left(\cos \omega X_{1}-\sin \omega X_{2}\right)\right. \\
\left.-\frac{\mathrm{I}}{\sqrt{E}} \frac{\partial \log \sqrt{\rho}}{\partial u}\left(\cos \theta X_{1}-\sin \theta X_{2}\right)\right] .
\end{array}\right.
$$

From these expressions we find that the direction-cosines $A_{1}, B_{1}, C_{1} ; A_{2}, B_{2}, C_{2}$ of the normals to the focal surfaces are given by

$$
\left\{\begin{array}{l}
A_{\mathrm{I}}=\frac{\mathrm{I}}{\sqrt{\mathrm{I}+\frac{\rho}{\rho_{1}}+2 \cos \sigma \sqrt{\frac{\rho}{\rho_{1}}}}}\left[\left(\cos \sigma+\sqrt{\frac{\rho}{\rho_{1}}}\right) X+\sin \sigma\left(\sin \theta X_{\mathrm{I}}-\cos \theta X_{2}\right)\right], \\
A_{2}=\frac{\mathrm{I}}{\sqrt{\mathrm{I}+\frac{\rho}{\rho_{1}}-2 \cos \sigma \sqrt{\frac{\rho}{\rho_{1}}}}}\left[\left(\cos \sigma-\sqrt{\frac{\rho}{\rho_{1}}}\right) X+\sin \sigma\left(\sin \theta X_{1}-\cos \theta X_{2}\right)\right] .
\end{array}\right.
$$

If $x_{0}, y_{0}, z_{0}$ are the coordinates of any point on the adjoint congruence, and we put

$$
P=\sum X\left(x-x_{0}\right), \quad Q=\sum\left(\sin \theta X_{1}-\cos \theta X_{2}\right)\left(x-x_{0}\right),
$$

where $x, y, z$ are current coordinates, the equations of planes tangent to $S$ and $S_{x}$ and of the focal planes are of the form

$$
P=0, \quad P \cos \sigma-Q \sin \sigma=0,
$$

$$
\left(\cos \sigma+\sqrt{\frac{\rho}{\rho_{1}}}\right) P+\sin \sigma Q=0, \quad\left(\cos \sigma-\sqrt{\frac{\rho}{\rho_{\mathrm{t}}}}\right) P+\sin \sigma Q=0 .
$$

Evidently the latter planes are harmonic conjugate with respect to the first two.

Consequently we have the theorem:

When $S$ and $S_{1}$ are two surfaces in the relation of a transformation $\mathbf{\Omega}$ their tangent planes are harmonic conjugate to the focal planes of the congruence generated by the line of intersection of these tangent planes.

From (84) it is seen that the necessary and sufficient condition that the focal planes be perpendicular is that $p_{1}=p$. Hence we have the theorem:

The necessary and sufficient condition that the lines of intersection of the tangent planes to the two surfaces $S$ and $S_{\mathrm{I}}$ in the relation of a transformation $\Omega$ form a normal congruence is that the associate surfaces $\mathbf{\Sigma}$ and $\mathbf{\Sigma}_{\mathrm{I}}$ bave equal tolal curvature at corresponding points; in this case the focal planes bisect the angles between the tangent planes to $S$ and $S_{1}$.

Bianchi has considered $W$-congruences of this kind ${ }^{\mathbf{r}}$ ); we shall apply the preceding formulas to this case.

${ }^{8}$ ) L. Branchl, Sopra alcune nuove classi di superficie e di sistemi tripli ortogonali [Annali di Ma. tematica pura ed applicata, Ser. II, Vol. XVIII (1890), pp. 301-358], pp. 330-334. 
$\$ 6$.

\section{Normal adjoint congruences.}

8. When $p_{\mathrm{I}}=\rho$, equations (I7) and (I8) may be replaced by

$$
\begin{gathered}
\frac{\partial \sigma}{\partial u}=\frac{\sin \sigma}{\mathrm{I}-\cos \sigma} \frac{\partial \log \rho}{\partial u}, \quad \frac{\partial \sigma}{\partial v}=-\frac{\sin \sigma}{\mathrm{I}+\cos \sigma} \frac{\partial \log \rho}{\partial v}, \\
\left\{\begin{array}{l}
\frac{\partial \log w_{1}}{\partial u}=\sqrt{E} \frac{\sin \sigma}{\mathrm{I}-\cos \sigma} \cos (\theta+\omega)-\frac{\partial \log \rho}{\partial u} \frac{\mathrm{I}}{\mathrm{I}-\cos \sigma}, \\
\frac{\partial \log w_{1}}{\partial v}=-\sqrt{G} \frac{\sin \sigma}{\mathrm{I}+\cos \sigma} \cos (\theta-\omega)-\frac{\partial \log \rho}{\partial v} \frac{\mathrm{I}}{\mathrm{I}+\cos \sigma} .
\end{array}\right.
\end{gathered}
$$

Moreover, equations (19) become

$$
\left\{\begin{array}{l}
\frac{\partial \theta}{\partial u}-A+\sqrt{E} \frac{\cos \sigma+\mathrm{I}}{\sin \sigma} \sin (\theta+\omega)=0 \\
\frac{\partial \theta}{\partial v}+B+\sqrt{G} \frac{\cos \sigma-\mathrm{I}}{\sin \sigma} \sin (\theta-\omega)=0 .
\end{array}\right.
$$

The condition of integrability of (87) is satisfied. The condition of integrability of $(85)$ is

or

$$
\frac{\partial^{2} \log \rho}{\partial u \partial v}+\frac{\partial \log p}{\partial u} \frac{\partial \log p}{\partial v}=0
$$

$$
\rho=\varphi(u)+\psi(v)
$$

In like manner the condition of integrability of (86) reduces to (88).

By means of (86) equations (26) become for the present case

$$
\left\{\begin{array}{l}
\sqrt{E} \sin (\theta+\omega)\left[\cot \left(\theta_{\mathrm{r}}+\omega_{\mathrm{I}}\right)+\cot (\theta+\omega)\right]=\frac{\mathrm{I}+\cos \sigma}{\sin \sigma} \frac{\partial \log p}{\partial u}, \\
\sqrt{G} \sin (\theta-\omega)\left[\cot \left(\theta_{\mathrm{r}}-\omega_{\mathrm{I}}\right)-\cot (\theta-\omega)\right]=\frac{\mathrm{I}-\cos \sigma}{\sin \sigma} \frac{\partial \log p}{\partial v},
\end{array}\right.
$$

equations which give $\theta_{1}$ and $\omega_{\mathrm{r}}$.

Since all the conditions of integrability of the foregoing equations are satisfied, we have the following theorems of BrancHI ${ }^{\text {rg }}$ ):

The necessary and sufficient condition that the curvature at corresponding points of the focal surfaces of a $W$-congruence be equal is that this curvature be of the form

$$
K=\frac{-1}{[\varphi(u)+\psi(v)]^{2}} .
$$

When the curvature of a surface $\mathbf{\Sigma}$ referred to its asymptotic lines is of the form (91), the determination of a surface $\Sigma_{1}$ referred to its asymptotic lines, whose curvature

19) L. Bianchi, loc. cit. ${ }^{\text {18}}$ ). 
is given by (9 $\mathrm{I})$ and such that $\mathbf{\Sigma}$ and $\mathbf{\Sigma}_{\mathrm{I}}$ are the focal surfaces of a $W$-congruence requires the solution of equations (85), (86) and (87), in which $p$ has the value (89).

The integral of equations $(85)$ is given by

where $k$ is a constant.

$$
\tan \frac{\sigma}{2}=\sqrt{\frac{\psi(v)-k}{\varphi(u)+k}},
$$

The surfaces $S$ whose associates possess this property are the only surfaces which can be deformed continuously with the preservation of a conjugate system. In fact, it is the conjugate system with the spherical representation determined by (89). Recalling the preceding results we have the following theorem:

If $S$ is a surface possessing a conjugate system which is preserved in a continuous deformation of $S$, there exist surfaces $S_{1}$ possessing the same property, determined by a solution of equations (87), in which $\sigma$ is given by (82), and by a quadrature (49), such that the lines joining corresponding points on $S$ and $S_{1}$ form a congruence whose developables meet $S$ and $S_{1}$ in these conjugate systems; moreover, the lines of intersection of the tangent planes to $S$ and $S_{1}$ form a normal congruence whose focal planes bisect the angles between the tangent planes to $S$ and $S_{1}$.

\section{$\int 7$}

\section{When $S$ and $S_{1}$ envelope a two-parameter family of spheres.}

9. In this section we consider the case where $S$ and $S_{I}$ are the envelope of a two-parameter family of spheres. In order that this be the case we must have

$$
x+r X=x_{1}+r X^{\prime}, \quad y+r Y=y_{1}+r Y^{\prime}, \quad z+r Z=z_{1}+r Z^{\prime},
$$

where $r$ denotes the radius of a sphere.

Substituting the values of $x_{1}$ and $X^{\prime}$ from (52) and (9), we are led to the three conditions

$$
\left\{\begin{array}{c}
R \sin 2 \omega(\mathrm{I}+\cos \sigma)=r \sin \sigma \\
R\left\{\cos \omega\left[\cot \left(\theta_{1}-\omega_{1}\right) \sin (\theta-\omega)-\cot \left(\theta_{\mathrm{r}}+\omega_{1}\right) \sin (\theta+\omega)\right]\right. \\
-\cos \sigma \sin \theta \sin 2 \omega\}+r \sin \sigma \sin \theta=0 \\
R\left\{\sin \omega\left[\cot \left(\theta_{1}-\omega_{1}\right) \sin (\theta-\omega)+\cot \left(\theta_{1}+\omega_{1}\right) \sin (\theta+\omega)\right]\right. \\
+\cos \sigma \cos \theta \sin 2 \omega\}-r \sin \sigma \cos \theta=0 .
\end{array}\right.
$$

Eliminating $r$ from the last two equations, we have

$$
\cot (\theta+\omega) \cot \left(\theta_{\mathrm{r}}-\omega_{\mathrm{r}}\right)-\cot (\theta-\omega) \cot \left(\theta_{1}+\omega_{\mathrm{l}}\right)=0 .
$$

Accordingly we introduce a function $\lambda$ by

$$
\cot \left(\theta_{1}-\omega_{1}\right)=\lambda \cot (\theta-\omega), \quad \cot \left(\theta_{1}+\omega_{1}\right)=\lambda \cot (\theta+\omega) .
$$

Substituting these values in the last two equations (94), multiplying by $\sin \theta$ and 
$-\cos \theta$ respectively and adding, we get

$R \sin 2 \omega(\lambda-\cos \sigma)+r \sin \sigma=0$.

From this and the first of (94), it follows that $\lambda=-$ I. Hence (95) become (96)

$$
\cot \left(\theta_{1}-\omega_{1}\right)=-\cot (\theta-\omega), \quad \cot \left(\theta_{1}+\omega_{s}\right)=-\cot (\theta+\omega) .
$$

In consequence of $(96)$ equations $(26)$ and $(28)$ reduce to

(97)

$$
\left\{\begin{array}{l}
\frac{\partial \log w_{1}}{\partial u}=\sqrt{E} \frac{\cos \sigma+\mathrm{I}}{\sin \sigma} \cos (\theta+\omega), \\
\frac{\partial \log w_{1}}{\partial v}=\sqrt{G} \frac{\cos \sigma+\mathrm{I}}{\sin \sigma} \cos (\theta-\omega),
\end{array}\right.
$$

and

$$
\left\{\begin{array}{l}
\frac{\partial \sigma}{\partial u}=\sqrt{E} \cos (\theta+\omega)\left(\mathrm{I}-\sqrt{\frac{\rho}{\rho_{\mathrm{I}}}}\right), \\
\frac{\partial \sigma}{\partial v}=\sqrt{G} \cos (\theta-\omega)\left(\mathrm{I}+\sqrt{\frac{\rho}{\rho_{\mathrm{I}}}}\right) .
\end{array}\right.
$$

The condition of integrability of equations (97) is reducible by means of (98) to

$$
\cos 2 \omega=0 \text {. }
$$

Hence the system on the sphere forms an orthogonal system from which it follows that $\Sigma$ is a minimal surface; also $S$ that is referred to its lines of curvature.

From (96) it follows that

$$
2 \omega_{1}=n \pi-2 \omega,
$$

where $n$ is on integer. Consequently $\cos 2 \omega_{1}=0$, which was to have been foreseen because of symmetry.

10. We shall find the equations for this case. From equations (3) follows the well-known fact that the system on the sphere is isothermic. We take

$$
\sqrt{E}=\sqrt{G}=e^{\alpha} .
$$

From equations (2) and the expressions for $\left\{\begin{array}{c}\mathrm{I} 2 \\ \mathrm{I}\end{array}\right\}^{\prime}$ and $\left\{\begin{array}{c}\mathrm{I} 2 \\ 2\end{array}\right\}^{\prime}$ it follows that

$$
\sqrt{p}=e^{-\alpha} \text {. }
$$

In like manner we write

$$
\sqrt{E}=\sqrt{G_{1}}=e^{a_{\mathrm{t}}}, \quad \sqrt{\hat{\rho}_{\mathrm{t}}}=e^{-\alpha_{\mathrm{i}}} .
$$

Equations (97) and (98) become

(I02) $\frac{\partial \log w_{1}}{\partial u}=e^{\alpha} \cos \left(\theta+\frac{\pi}{4}\right) \frac{\cos \sigma+1}{\sin \sigma}, \frac{\partial \log w_{1}}{\partial v}=e^{\alpha} \cos \left(\theta-\frac{\pi}{4}\right) \frac{\cos \sigma+1}{\sin \sigma}$,

(103) $\frac{\partial \sigma}{\partial u}=\cos \left(\theta+\frac{\pi}{4}\right)\left(e^{\alpha}-e^{\alpha_{1}}\right), \quad \frac{\partial \sigma}{\partial v}=\cos \left(\theta-\frac{\pi}{4}\right)\left(e^{\alpha}+e^{\alpha_{1}}\right)$. 
Also equations (29) reduce to

(I04)

$$
\left\{\begin{array}{l}
\frac{\partial}{\partial u}\left(\alpha_{1}+\alpha\right)=\cos \left(\theta+\frac{\pi}{4}\right)\left(e^{\alpha}-e^{\alpha_{1}}\right) \frac{\cos \sigma+1}{\sin \sigma}, \\
\frac{\partial}{\partial v}\left(\alpha_{1}+\alpha\right)=\cos \left(\theta-\frac{\pi}{4}\right)\left(e^{\alpha}+e^{\alpha_{1}}\right) \frac{\cos \sigma+\mathrm{I}}{\sin \sigma} .
\end{array}\right.
$$

Equations (103) and (104) may be combined so that we have by integration

$$
\sin \frac{\sigma}{2}=c e^{\frac{\alpha+\alpha_{\mathrm{I}}}{2}}
$$

where $c$ denotes a constant.

Furthermore, equations (19) assume that form

$$
\left\{\begin{array}{l}
\sin \sigma\left(\frac{\partial \theta}{\partial u}-\frac{\partial \alpha}{\partial v}\right)+\left(e^{\alpha} \cos \sigma+e^{\alpha_{1}}\right) \sin \left(\theta+\frac{\pi}{4}\right)=0 \\
\sin \sigma\left(\frac{\partial \theta}{\partial v}+\frac{\partial \alpha}{\partial u}\right)+\left(e^{\alpha} \cos \sigma-e^{\alpha_{1}}\right) \sin \left(\theta-\frac{\pi}{4}\right)=0 .
\end{array}\right.
$$

Equations (103), (104), (106) determine a transformation of ThyBaUT ${ }^{20}$ ) of minimal surfaces.

If we put

$$
\varphi=e^{-\left(\alpha_{1}+\alpha\right)} w_{\mathrm{s}},
$$

it follows from (102) and (104) that

$$
\text { (108) } \frac{\partial \log \varphi}{\partial u}=\cos \left(\theta+\frac{\pi}{4}\right) e^{\alpha_{1}} \frac{\cos \sigma+\mathrm{I}}{\sin \sigma}, \frac{\partial \log \varphi}{\partial v}=-\cos \left(\theta-\frac{\pi}{4}\right) e^{x_{1}} \frac{\cos \sigma+\mathrm{I}}{\sin \sigma} \text {. }
$$

Again from (102), (107) and (108) it follows that

$$
\frac{\partial \varphi}{\partial u}=e^{-2 \alpha} \frac{\partial w_{s}}{\partial u}, \quad \frac{\partial \varphi}{\partial v}=-e^{-2 \alpha} \frac{\partial w_{1}}{\partial v} .
$$

We have observed that in the present case the surfaces of which $\boldsymbol{\Sigma}$ and $\boldsymbol{\Sigma}_{\mathrm{f}}$ are associate have their lines of curvature represented on the sphere by an isothermic orthogonal system, and conversely all such surfaces $S$ have a minimal surface for associate. Hence we have a transformation of such surfaces $S$ possessing the property that $S$ and a transform form the envelope of a two parameter family of spheres. When the functions $\theta, \alpha_{1}$ and $\sigma$ are known, the further determination of $S_{\mathrm{r}}$ requires the integration of equations (49) which in consequence of (107) may be given the form

$$
\frac{\partial}{\partial u}\left(\varphi W_{1}\right)=-e^{-2 x} w_{\mathrm{I}}^{2} \frac{\partial}{\partial u}\left(\frac{W}{w_{\mathrm{I}}}\right), \quad \frac{\partial}{\partial v}\left(\varphi W_{\mathrm{l}}\right)=e^{-2 \alpha} w_{\mathrm{s}}^{2} \frac{\partial}{\partial v}\left(\frac{W}{w_{\mathrm{s}}}\right) .
$$

20) A. ThyвaUt, Sur la deformation du paraboloide et sur quelques problèmes qui s'y rattachent [Annales Scientifiques de l'École Normale supérieure (Paris), Ser. III, Vol. XIV, pp. 45.98; cfr. also: L. Bianchi, loc. cit. ${ }^{3}$ ), pp. 334-338. 
These are the same transformations which we discovered in a former paper ${ }^{21}$ ) by analytical considerations, but now their bearing upon the general problems of transformations of conjugate systems with equal tangential invariants is clearly in evidence.

Princeton University, March 7, 19I4

Luther Pfahler EISENhart.

21) L. P. EIsEnhart, Surfaces with isolbermal representation of their lines of curvature and their transformations [Transactions of the American Mathematical Society, Vol. IX (1908), pp. 149-177]. 